\title{
The eDAL Suite: Tools and Concepts for Primary Data Citation
}

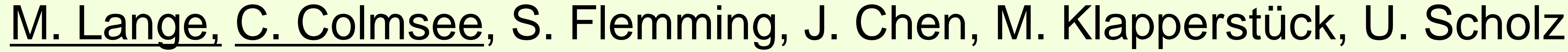

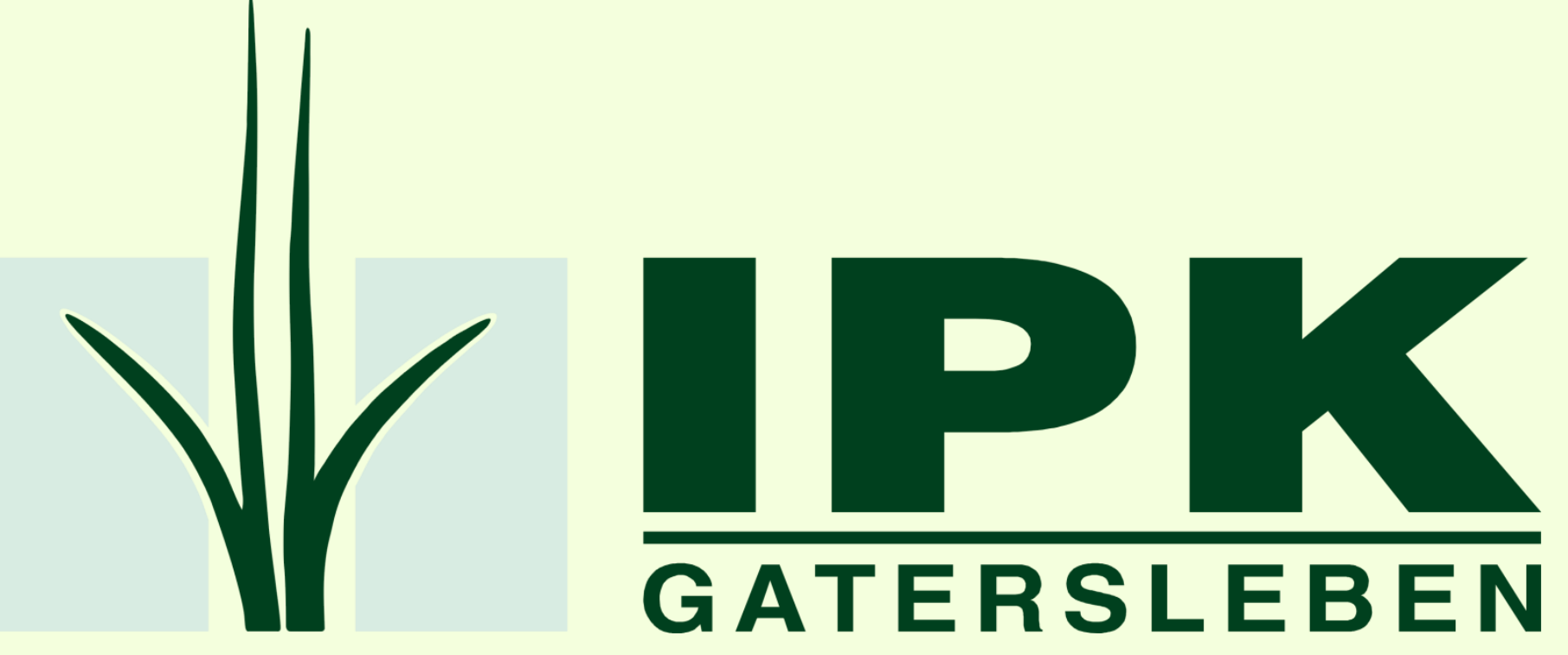

Research Group Bioinformatics and Information Technology,

Leibniz Institute of Plant Genetics and Crop Plant Research (IPK), Corrensstraße 3, 06466 Gatersleben, Germany

Contact: <lange>|<colmsee>@ipk-gatersleben.de

\section{Motivation}

Retrieval and citation of primary data is the important factor in the approaching age of "data science". Digital data are easily shared, and just as easily wiped or lost. The problem of keeping on-line data accessible and retrievable is especially difficult for SME like plant breeders plant biotech companies as well as research projects in this domain.

Intension of eDAL is the provisioning of an information retrieval and data citation infrastructure that meets the requirements of the "data science" age and implements a re-usable platform for data retrieval, data citation, and data publication. Like a shopping cart, the idea is to combine a search engine and a data cart, which retrieves, rank and collect query relevant data from crop plant data centers.

Distributed, Redundant, Alternative Data @ IPK: 916 data sets for „phosphate ransporter“ in 4 IPK databases

CR-EST

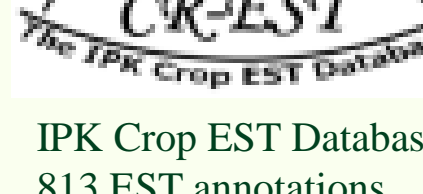

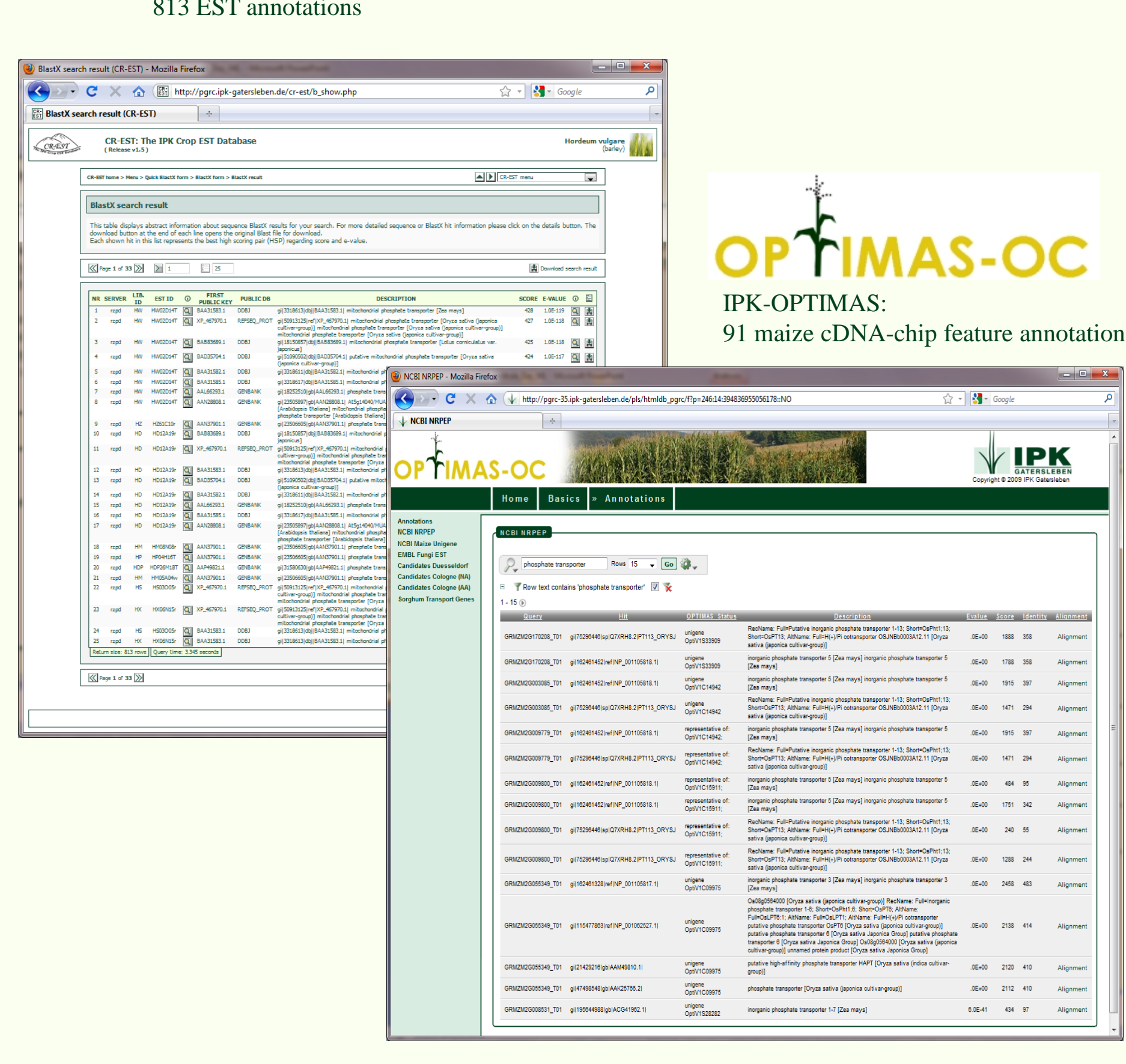

POCI

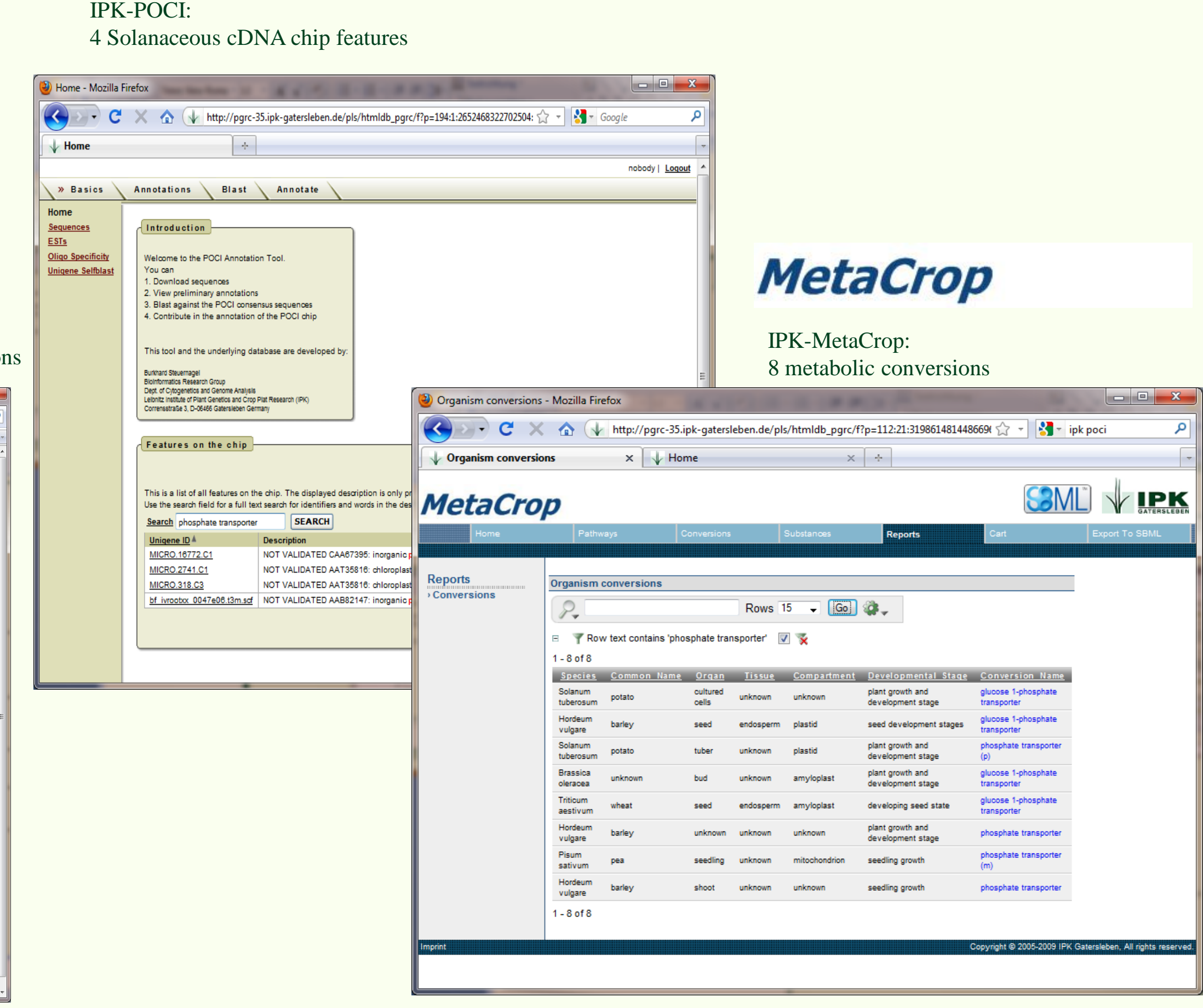

Availability of Data

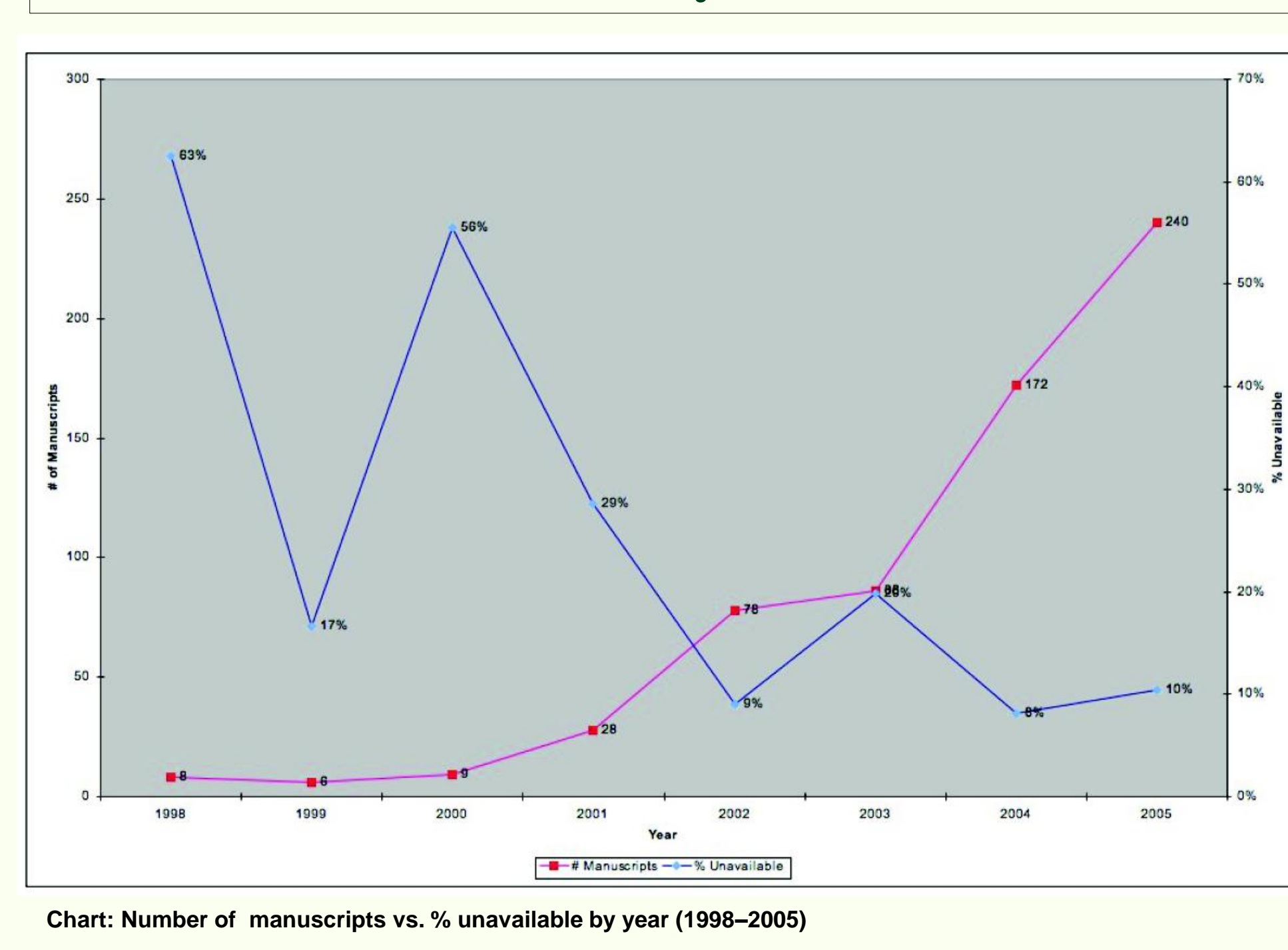

The increasing number of digital data should lead into a long-term storage system, within the data is available at all time. But Anderson et al. illustrates the problematic of the loss of digital data within edical publications. The reported up to $30 \%$ unavailability of supplementary data in 9 years.
Data Citation Rate

The importance of sharing data within publications is shown in Piwowar et al. for microarray data. Publications comprising shared ata have a $69 \%$ higher citation ate than publications without shared data.

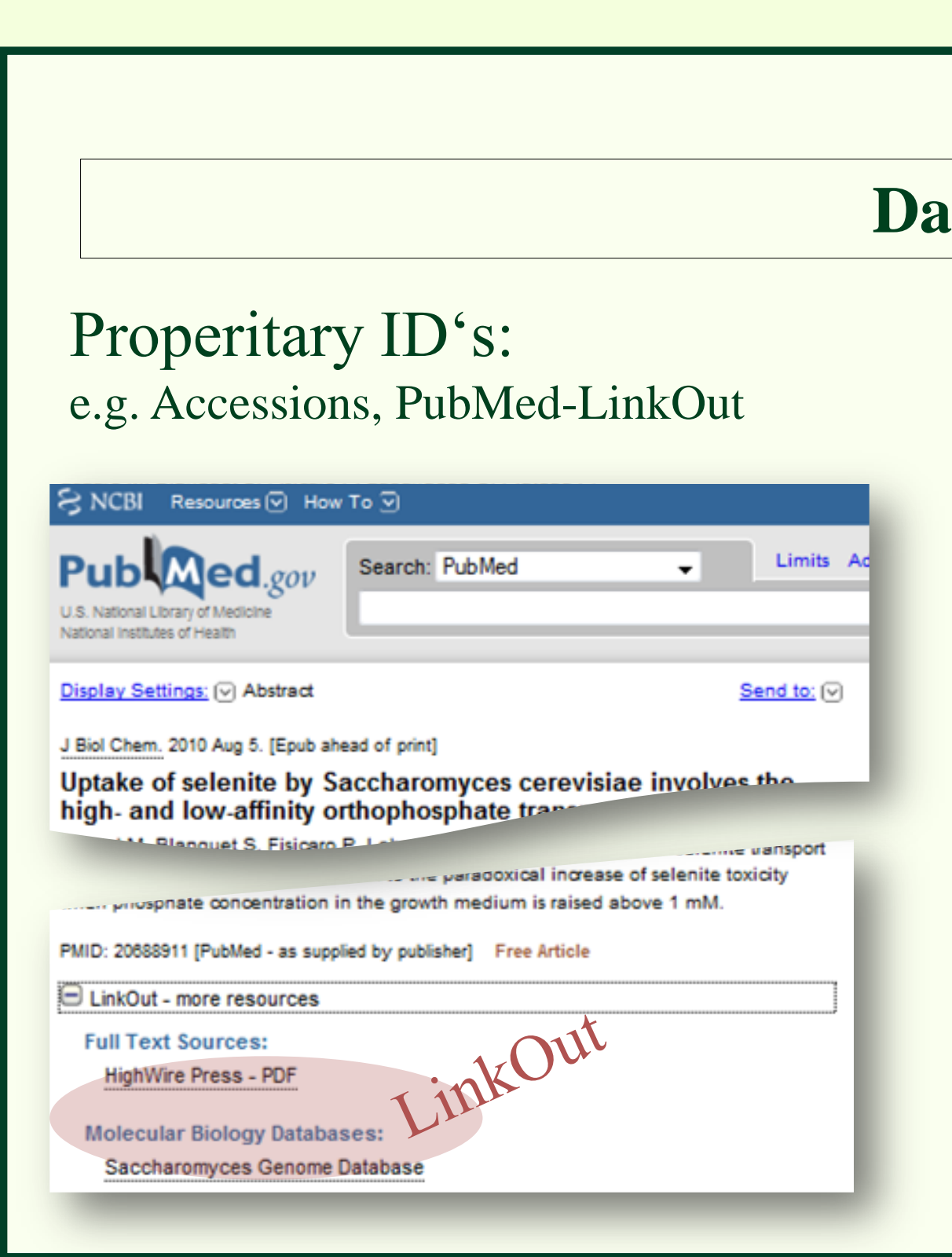

\section{State of the Art}

Data Citation

$$
\text { Standard ID‘s: }
$$

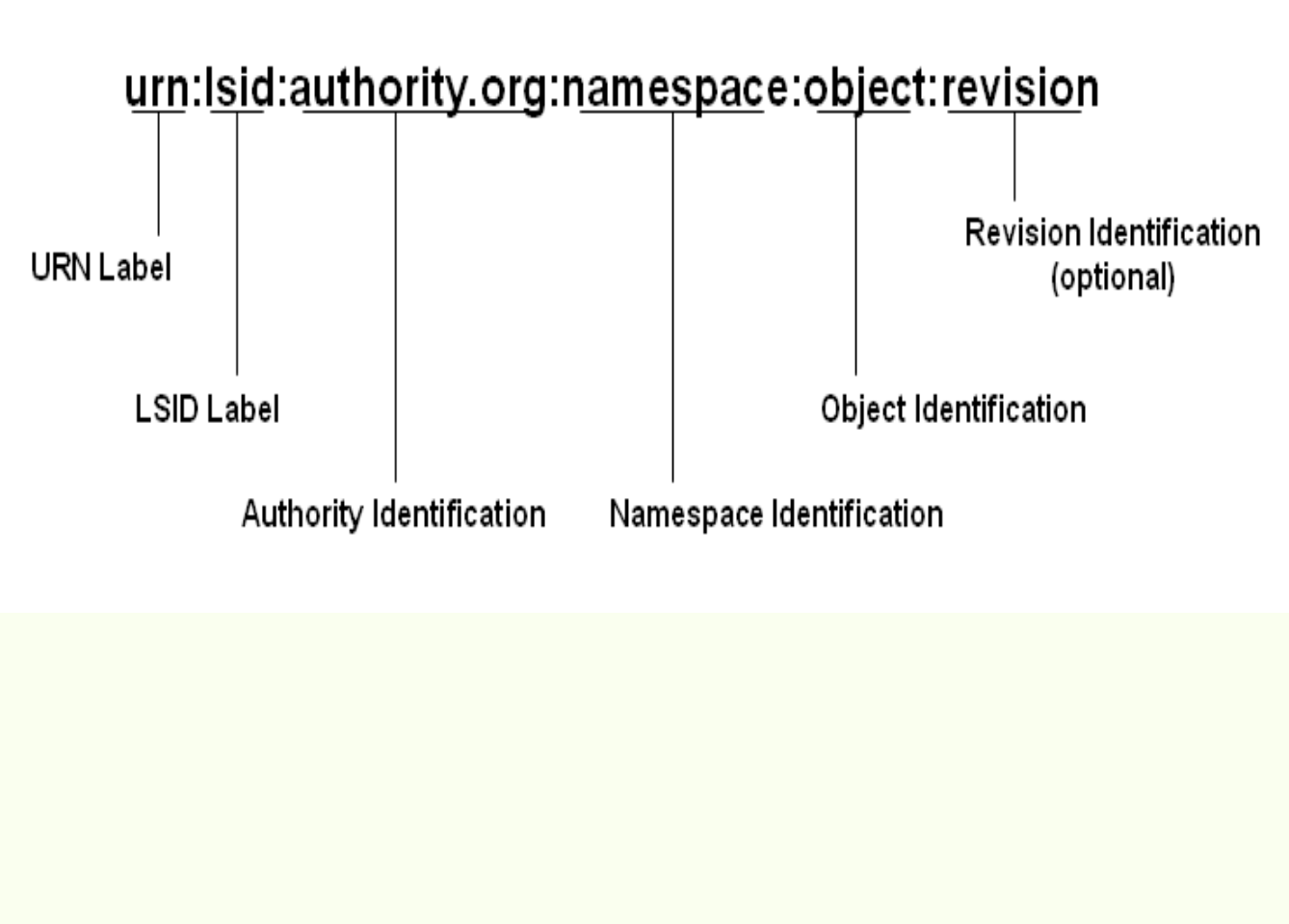

Meta Data

Technical meta data: Semantic meta data: e.g. ISO 15836 (DCMES) e.g. EBI Ontology Lockup Service

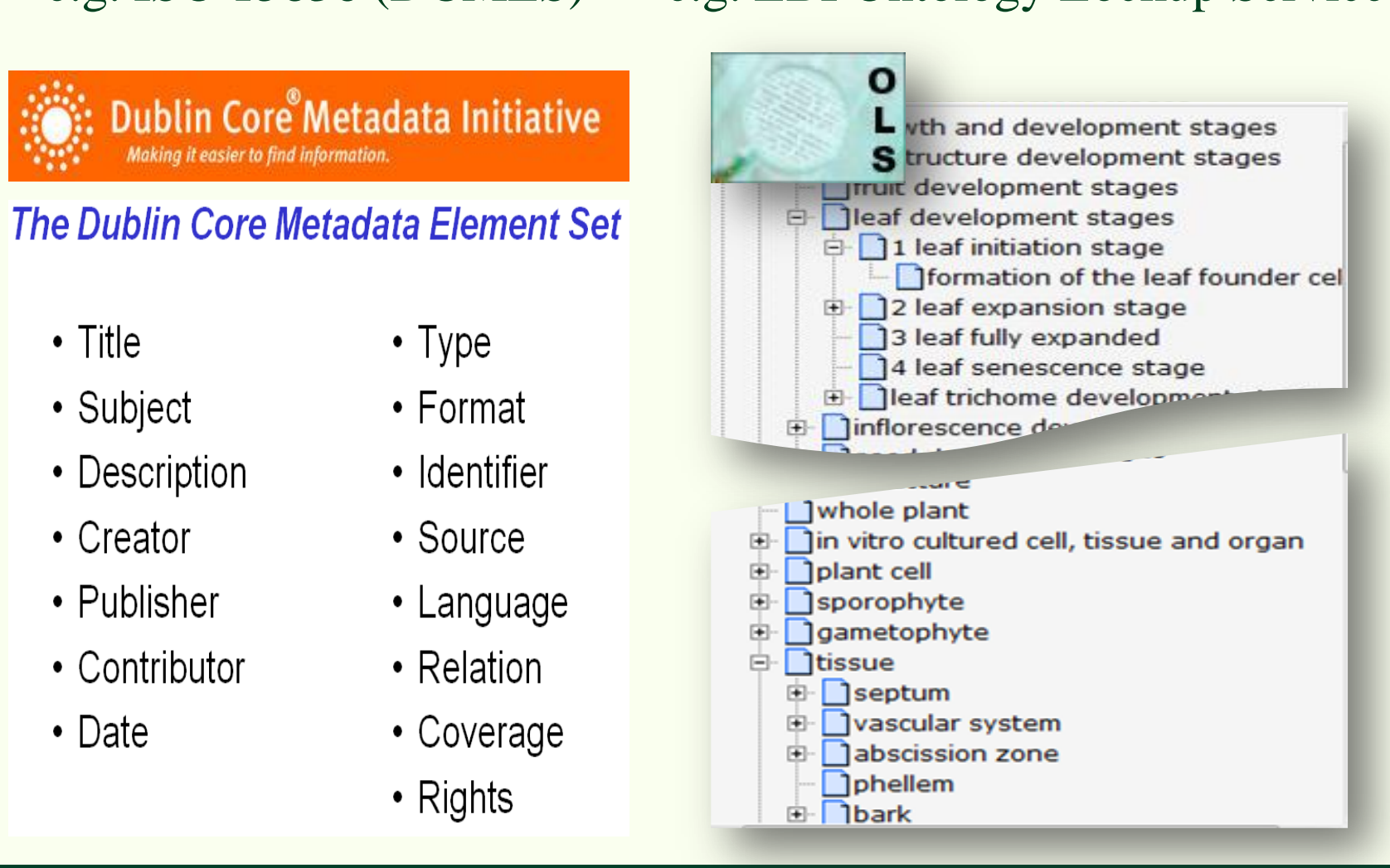

Data Access

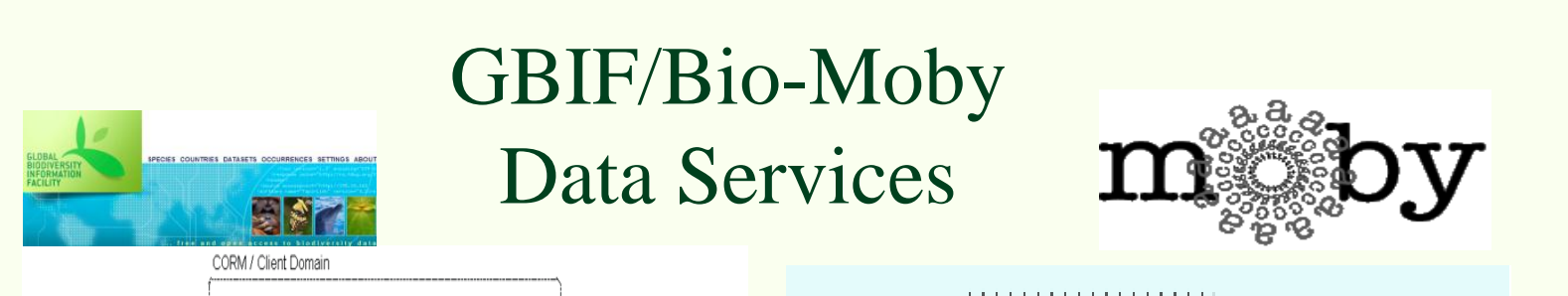

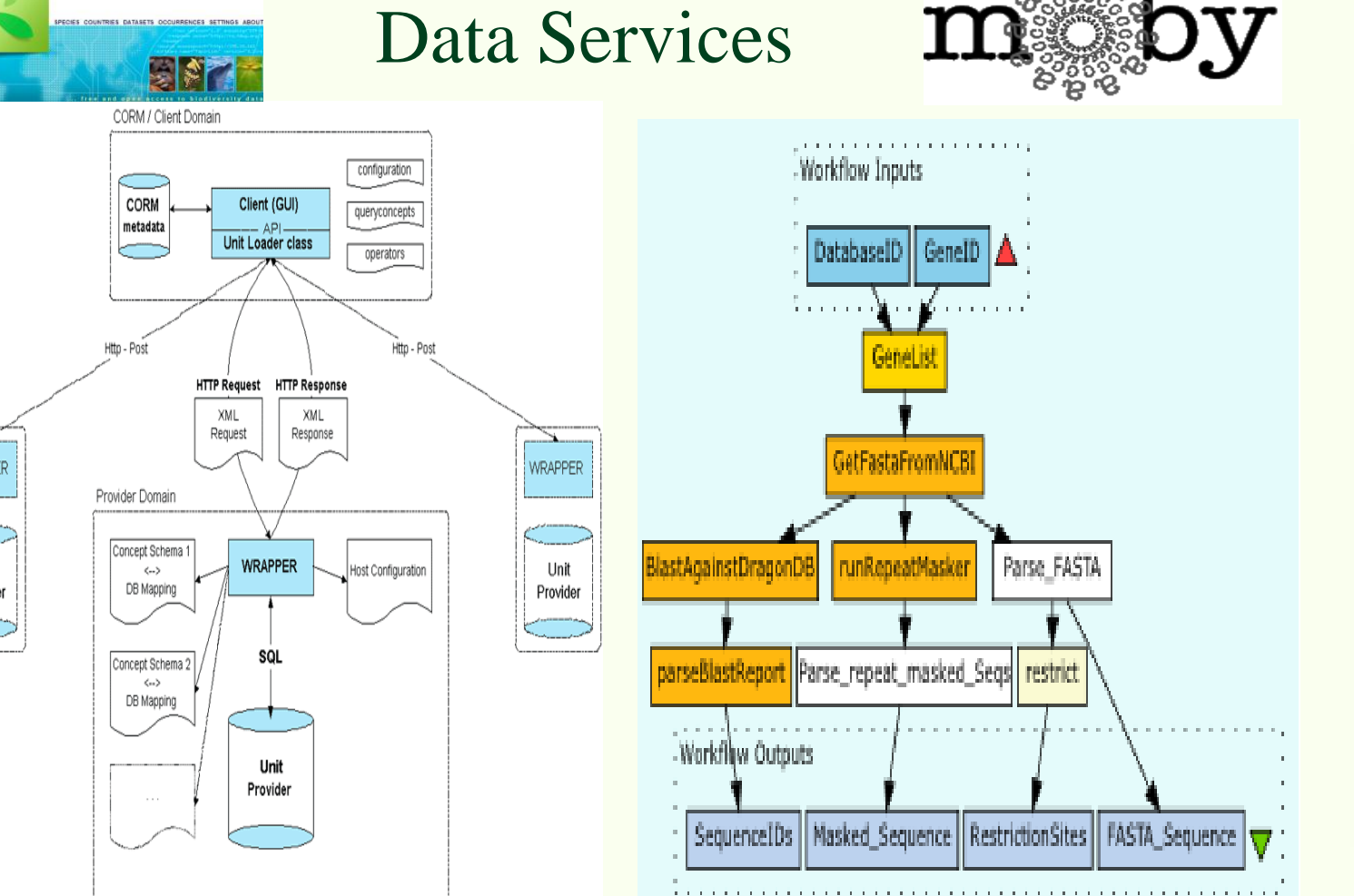

Search Engine EBI EB-Eye/NCBI Entrez

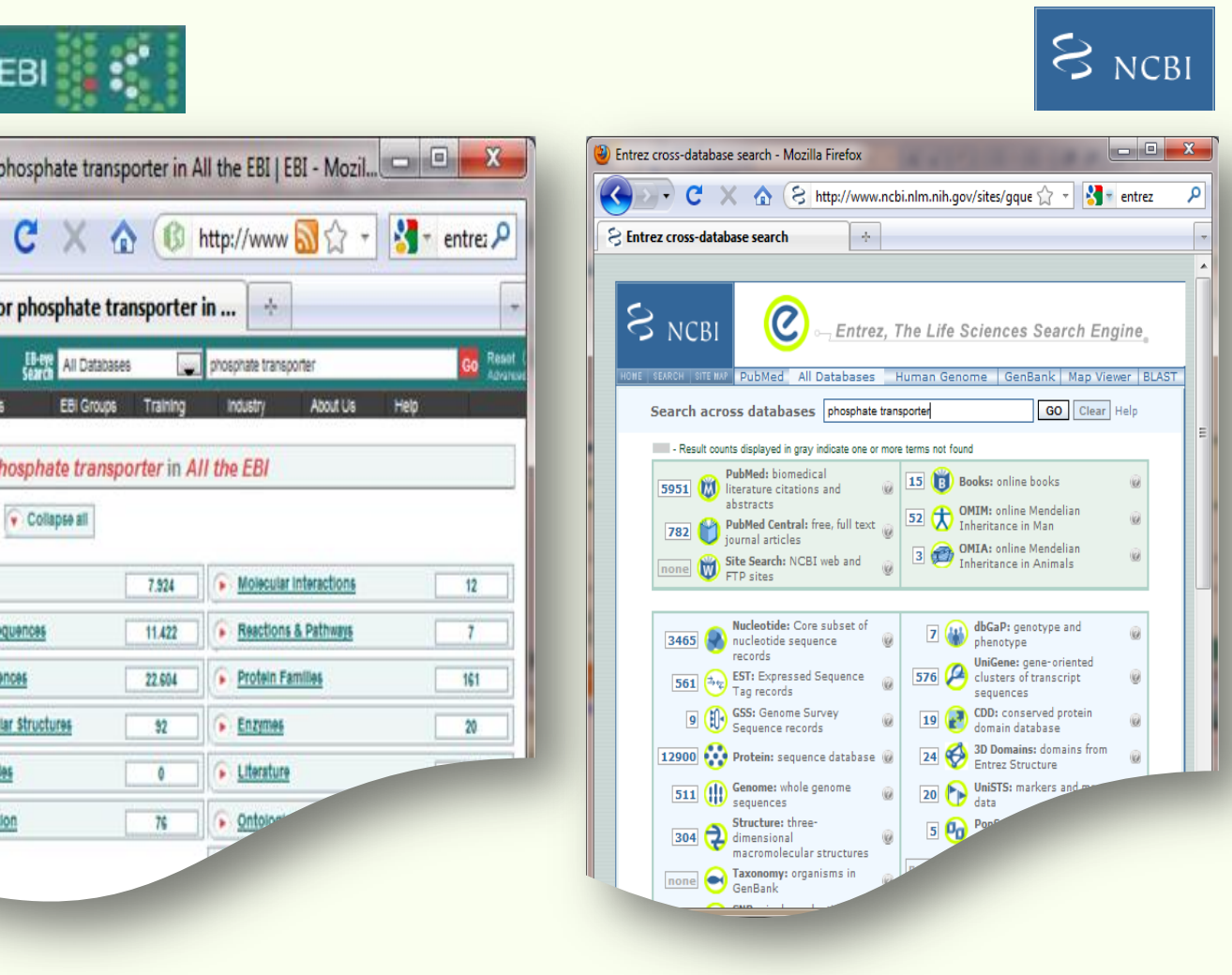

\section{Primary Data Management at the IPK}

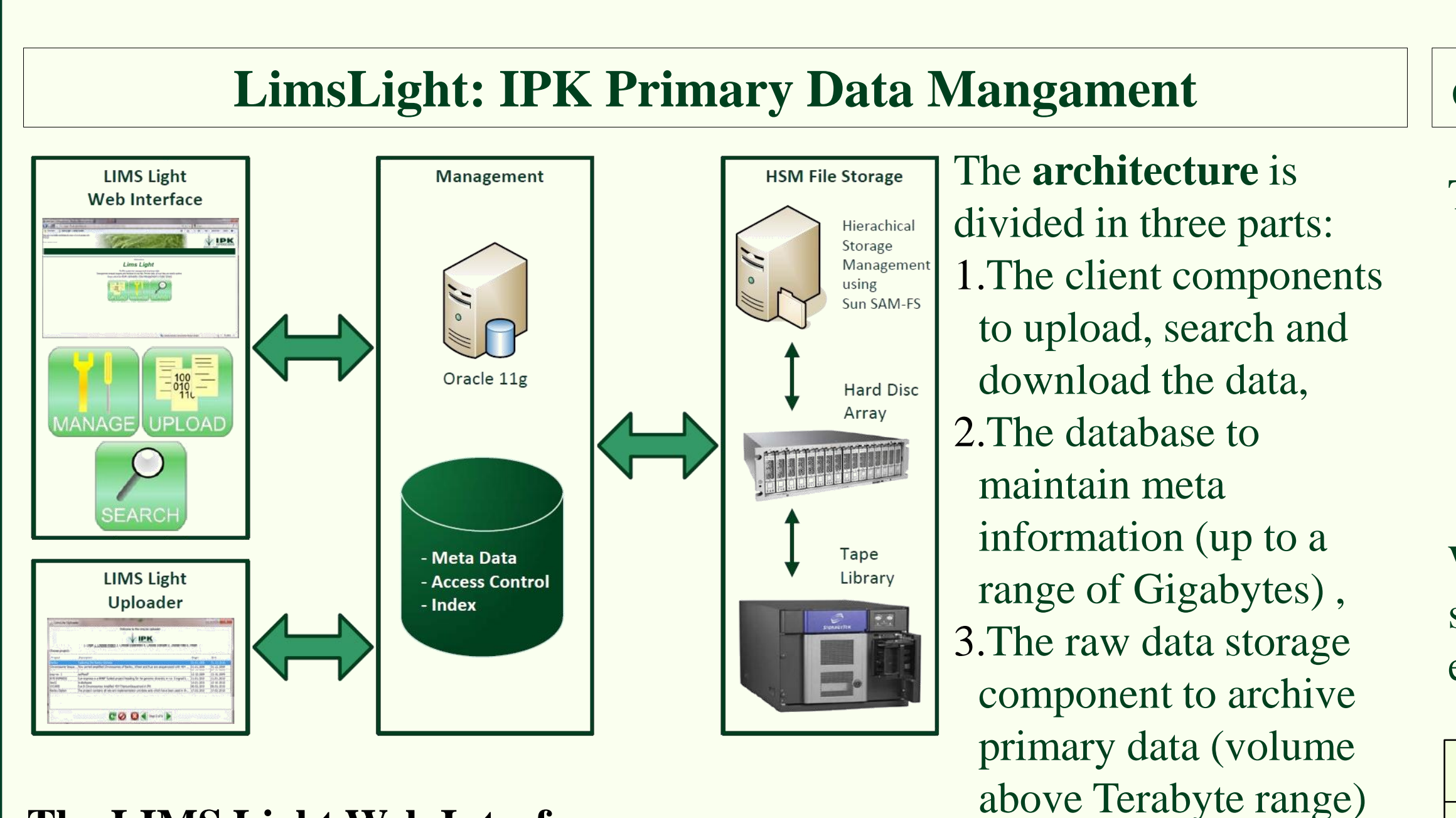

eDAL-API: Interface to Store, Manage and Annotate Primary Data

The eDAL-API takes care of the central aspects of primary data management:

Storage component to archive the primary data

Persistent identifier to make primary data citab

- JAAS security for data access control

Versioning of primary data

With the eDAL-API the user can implement his own Primary Data Management ystem and could realize the information retrieval by using the life science searc

The LIMS Light Web Interfaces

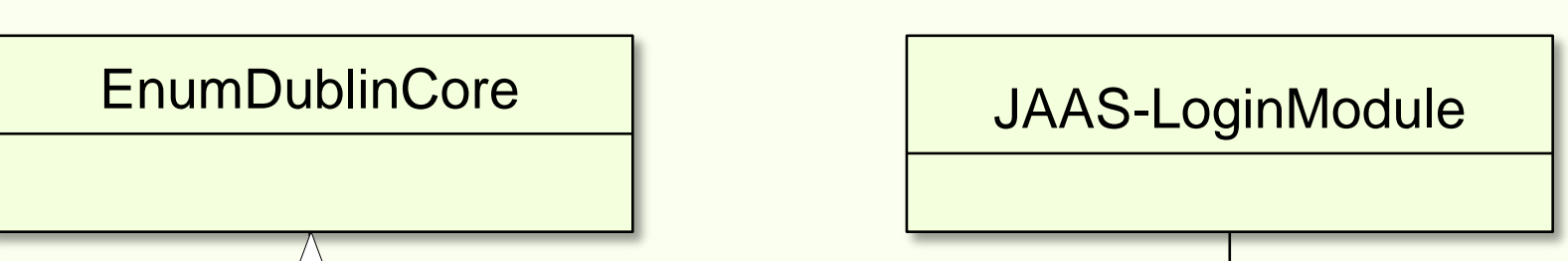

Web application based on ORACLE Application Express (APEX)

Management of Projects, Experiments, Worksets and files

Using controlled vocabulary

Simple and advanced search to find stored files
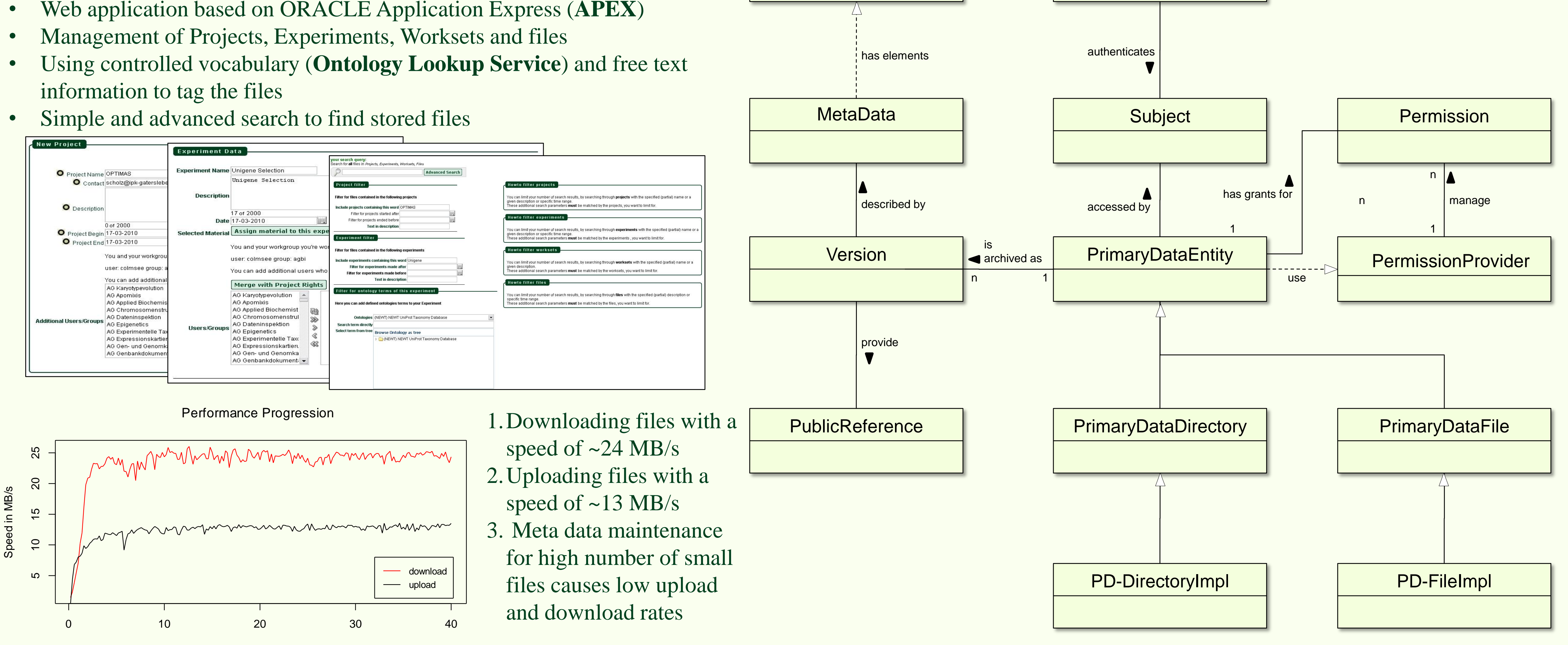

Outlook: The CROP-SHOP approach (submitted as BMBF proposa) Data centers and primary data archives (1) provide access to their databases and information systems by a uniform data citation system. The information retrieva component is featured by a search engine (2) and a data cart (3). The search engine retrieves data, which are ranked for their query and user specific relevance profiles. The linked to administrative global object identifiers resulting from a query. The identifiers are processing (4) in adequate data formats.

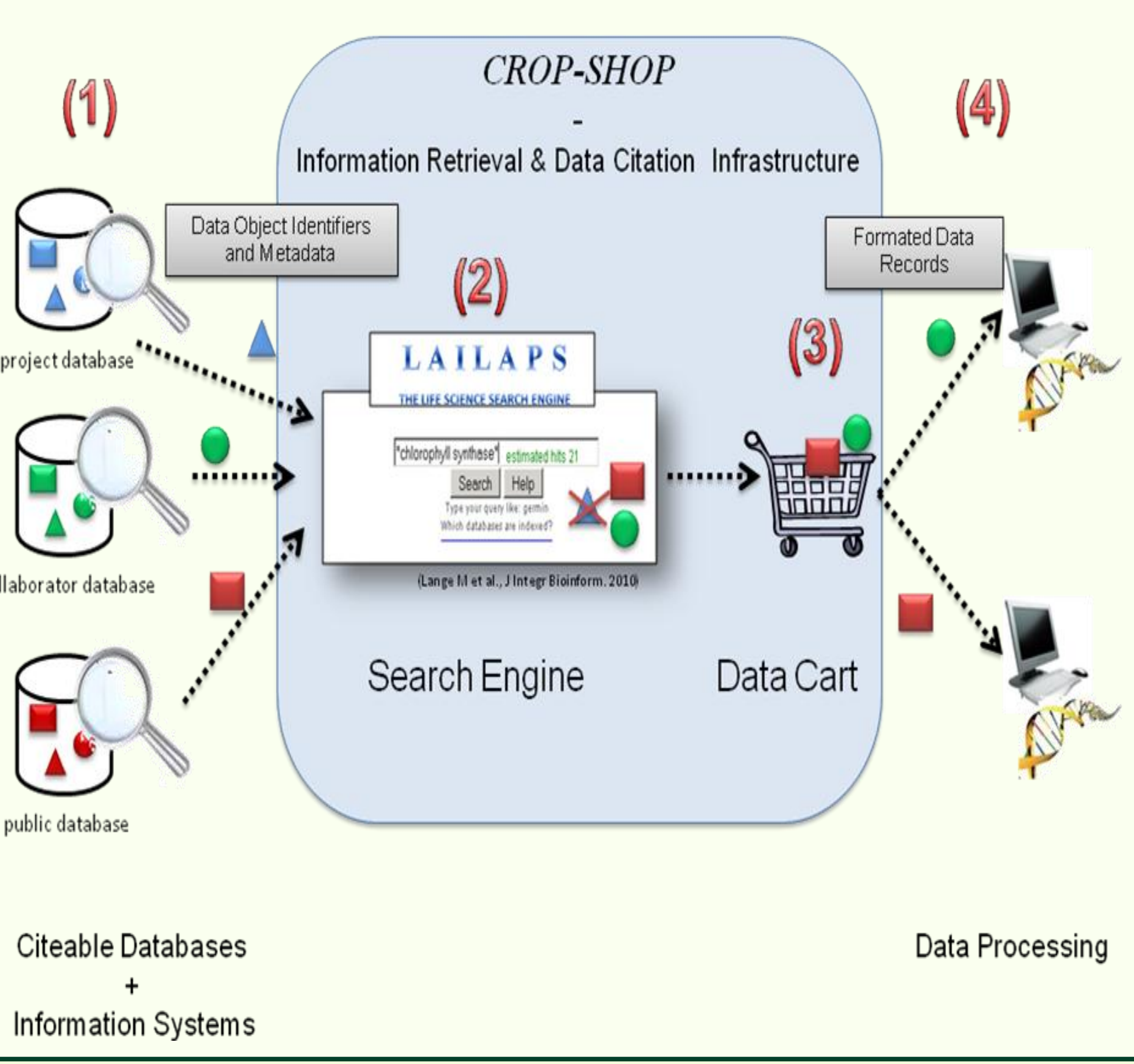

